\title{
Pion in a uniform background magnetic field with clover fermions
}

\author{
Ryan Bignellø, Waseem Kamleh๑, and Derek Leinweber@ \\ Special Research Centre for the Subatomic Structure of Matter (CSSM), Department of Physics, \\ University of Adelaide, Adelaide, South Australia 5005, Australia
}

(Received 1 November 2019; published 31 December 2019)

\begin{abstract}
Background-field methods provide an important nonperturbative formalism for the determination of hadronic properties which are complementary to matrix-element calculations. However, new challenges are encountered when utilizing a fermion action exposed to additive mass renormalizations. In this case, the background field can induce an undesired field-dependent additive mass renormalization that acts to change the quark mass as the background field is changed. For example, in a calculation utilizing Wilson fermions in a uniform background magnetic field, the Wilson term introduced a field-dependent renormalization to the quark mass which manifests itself in an unphysical increase of the neutral-pion mass for large magnetic fields. Herein, the clover-fermion action is studied to determine the extent to which the removal of $\mathcal{O}(a)$ discretization errors suppresses the field-dependent changes to the quark mass. We illustrate how a careful treatment of nonperturbative improvement is necessary to resolve this artifact of the Wilson term. Using the $32^{3} \times 64$ dynamical-fermion lattices provided by the PACS-CS Collaboration we demonstrate how our technique suppresses the unphysical mass renormalization over a broad range of magnetic-field strengths.
\end{abstract}

DOI: 10.1103/PhysRevD.100.114518

\section{INTRODUCTION}

The uniform background-field method [1-3] has a long history of use in lattice QCD to investigate and calculate quantities such as hadronic magnetic moments [2,4-6], polarizabilities [4,6-9], and the spatial distribution of quarks in a magnetic field [10]. Recently, it has been demonstrated that the background-field method introduces unphysical changes in the fermion energy when the Wilson quark formulation is used [11,12].

Bali et al. highlighted this important problem [11,12] arising from the Wilson-fermion action with the background-field method. In particular they determined that in the free-field limit, the mass of a Wilson quark is shifted by an amount $\frac{a}{2}|q e B|$ to

$$
m_{[\mathrm{w}]}(B)=m_{\mathrm{w}}(0)+\frac{a}{2}|q e B|,
$$

where $a$ is the lattice spacing, $B$ is the magnetic-field strength, $q e$ is the quark charge, and $m_{\mathrm{w}}$ is the Wilson quark mass. Here we have introduced the notation that a subscript label in square brackets represents a quantity that

*ryan.bignell@adelaide.edu.au

Published by the American Physical Society under the terms of the Creative Commons Attribution 4.0 International license. Further distribution of this work must maintain attribution to the author(s) and the published article's title, journal citation, and DOI. Funded by SCOAP. is affected by lattice background-field artifacts due to the fermion action, in this case the Wilson quark mass $m_{[\mathrm{w}]}(B)$. This notation will be used throughout this work in order to distinguish between energy values with and without the additive background-field mass renormalization, respectively [e.g., to distinguish the pion mass $m_{[\pi]}(B)$ and $m_{\pi}$ ].

It was shown in Refs. $[8,11]$ that the overlap quark formalism $[13,14]$ does not suffer from this problem of field-dependent mass renormalization. As the overlap action is many times more computationally expensive than the Wilson action, we turn our focus to the Wilson cloverfermion action to determine its suitability with respect to the additive mass renormalization arising from the background field.

The clover-fermion action [15] is designed to remove $\mathcal{O}(a)$ lattice artifacts arising from the Wilson term. Thus, it is interesting to examine the extent to which the Wilsonfermion artifacts survive in the clover-fermion formulation. Herein, the clover fermion is studied in both the free-field limit and full QCD to determine its efficacy in removing the unphysical energy change caused by the uniform background magnetic field.

Pion correlation functions are the natural choice with which to investigate this issue; they are free of complications associated with the magnetic moment, provide precision at low computational cost, and offer insight into interesting physics through the difference between neutral and charged pions. The neutral pion is of particular interest as it also has no hadronic-level Landau contribution. 
The free-field limit allows the unphysical additive mass renormalization to be studied, without the complications of QCD. This aids in formulating a solution for this problem using clover fermions. When full QCD interactions are present, the competing effects of QCD and the background magnetic field make isolating and understanding the additive mass renormalization more challenging. Moreover, the use of a nonperturbatively improved clover coefficient in a full QCD calculations further complicates the transition from free-field to full QCD interactions.

Here, the clover-fermion action is studied for both the free-field and full-QCD cases to determine the conditions for the removal of the field-dependent additive quark-mass renormalization. In Sec. II a brief overview of the background-field method is presented while Sec. III details the calculations performed in the free-field limit. These confirm the presence of the additive mass renormalization and the utility of the clover-fermion action in this limit. Full QCD is considered in Sec. IV and Sec. V details how the clover-fermion action can be tuned to remove the fielddependent additive quark-mass renormalization. The magnetic polarizability of the neutral pion is presented in Sec. V B and conclusions are summarized in Sec. VI.

\section{BACKGROUND-FIELD METHOD}

In this approach, a constant background magnetic field is introduced and the eigenstates are examined in the basis of the full Hamiltonian [3]. We commence in the continuum where a minimal electromagnetic coupling is added to form the covariant derivative

$$
D_{\mu}=\partial_{\mu}+i q e A_{\mu} .
$$

Here $q e$ is the electric charge of the fermion field and $A_{\mu}$ is the electromagnetic four potential. On the lattice, the equivalent modification is to multiply the QCD gauge links by an exponential phase factor

$$
U_{\mu}(x) \rightarrow U_{\mu}(x) e^{\text {iaqeA }_{\mu}(x)} .
$$

To generate a uniform magnetic field along the $\hat{z}$ axis in the continuum, one considers

$$
\begin{gathered}
\vec{B}=\nabla \times \vec{A}, \\
B_{z}=\partial_{x} A_{y}-\partial_{y} A_{x} .
\end{gathered}
$$

This does not uniquely specify the electromagnetic potential. The choice commonly selected over the interior of the lattice is $A_{x}=-B y$ which gives a constant magnetic field of magnitude $B$ in the $+\hat{z}$ direction. Due to the periodic boundary conditions on the lattice, we set $A_{y}=+B N_{y} x$ along the boundary in the $\hat{y}$ dimension to maintain the constant magnetic field across the boundary. Now, considering the entirety of the lattice, a quantization condition [1] for the magnetic-field strength is required:

$$
q e B a^{2}=\frac{2 \pi k_{B}}{N_{x} N_{y}}
$$

where $a$ is the lattice spacing, $N_{x}$ and $N_{y}$ are the spatial dimensions of the lattice and $k_{B}$ is an integer specifying the field strength in terms of the minimum field strength.

In this work the field quanta $k_{B}$ is in terms of the charge of the down quark, i.e., $q=-1 / 3$ such that

$$
e B=\frac{-6 \pi k}{a^{2} N_{x} N_{y}} .
$$

Hence a field with $k_{B}=1$ will be in the $-\hat{z}$ direction.

\section{FREE-FIELD CASE}

The free-field simulation is investigated first; here the quarks couple to the external magnetic field through their electric charges but do not experience any QCD effects. The energy of such a charged particle contains a Landau energy term proportional to the charge of the particle. In the nonrelativistic approximation with $\vec{B}=B \hat{z}$, this energy spectrum is equivalent to that of a harmonic oscillator:

$$
E=\left(n+\frac{1}{2}\right) \frac{|q e B|}{m}, \quad n=0,1, \ldots
$$

The relativistic generalization of the Landau energy applies to each fermion responding to the field. For a free quark, the energy is

$$
E^{2}(B)=m^{2}+(2 n+1)|q e B|+p_{z}^{2}+2 \vec{s} \cdot q e \vec{B} .
$$

Here $p_{z}$ is the quark momentum in the $\hat{z}$ direction, $|\vec{s}|=$ $1 / 2$ and the quark has charge qe.

\section{A. Wilson fermions}

Wilson fermions will have an additional energy term according to Eq. (1), and thus a free-field energy

$$
\begin{aligned}
E_{[\mathrm{w}]}^{2}(B)= & \left(m_{\mathrm{w}}+\frac{a}{2}|q e B|\right)^{2} \\
& +(2 n+1)|q e B|+p_{z}^{2}+2 \vec{s} \cdot q e \vec{B},
\end{aligned}
$$

as the Wilson term is the discretized lattice Laplacian which also describes the lowest-lying Landau level [9].

As $a$ is the lattice spacing, this additional term is absent in the continuum limit. The additional term in Eq. (9) is a lattice artifact identified with field-strength-dependent additive quark-mass renormalization.

To demonstrate the presence of this additive mass term, the free-field pion mass is calculated. Here we consider 
both the charged pion energy $E_{\pi^{ \pm}}$and the neutral connected pion energy $E_{\pi_{u / d}^{0}}(B)$ and focus on the lowest-lying states. Standard pseudoscalar interpolating fields $\chi=\bar{q} \gamma_{5} q$ are considered, where the quark flavors are either $u \bar{u}$ or $d \bar{d}$ corresponding to $\pi_{u}^{0}$ and $\pi_{d}^{0}$.

For a neutral pion with quark content $u \bar{u}$ or $d \bar{d}$ it is possible to have the spin-dependent term of Eq. (9), $2 \vec{s} \cdot q e \vec{B}$, cancel the Landau energy term $(2 n+1)|q e B|$ for $n=0$. This cancellation of terms occurs for both the quark and the antiquark. Consider, for example, the $u \bar{u}$ pseudoscalar. As a spin-zero state, the two quarks have opposite spin orientations. Similarly, the quark and antiquark have opposite electric charges. If the $u$ quark is spin down and the $\bar{u}$ is spin up, the terms cancel. For $p_{z}=0$, one has the lowest-lying state with energy

$$
\begin{aligned}
E_{\left[\pi^{0}\right]}(B) & =E_{[u]}(B)+E_{[\bar{u}]}(B) \\
& =m_{u}+\frac{a}{2}\left|q_{u} e B\right|+m_{\bar{u}}+\frac{a}{2}\left|q_{\bar{u}} e B\right| \\
& =m_{\pi^{0}}+a\left|q_{u} e B\right| .
\end{aligned}
$$

For a charged pion the spin-dependent term cannot cancel the Landau term for both the quark and antiquark sectors. The lowest-energy state is realized when the terms cancel for the quark flavor with the largest magnitude of electric charge.

Consider, for example, the $\pi^{+}$meson composed of $u \bar{d}$. Here the lowest-energy state is realized when the $u$ quark is spin down, enabling cancellation of the spin-dependent and Landau terms. For $p_{z}=0$, the charged pion will hence have energy

$$
\begin{aligned}
E_{\left[\pi^{+}\right]}(B)= & E_{[u]}(B)+E_{[\bar{d}]}(B) \\
= & m_{u}+\frac{a}{2}\left|q_{u} e B\right| \\
& +\sqrt{\left(m_{\bar{d}}+\frac{a}{2}\left|q_{\bar{d}} e B\right|\right)^{2}+2\left|q_{\bar{d}} e B\right| .}
\end{aligned}
$$

Note that as QCD interactions are not present, no energy is required to displace the quarks from each other and thus the magnetic polarizability vanishes.

In the absence of the Wilson background-field additive mass renormalization, the charged pion energy becomes

$$
\begin{aligned}
E_{\pi^{+}}(B) & =E_{u}(B)+E_{\bar{d}}(B) \\
& =m_{u}+\sqrt{m_{\bar{d}}^{2}+2\left|q_{\bar{d}} e B\right| .}
\end{aligned}
$$

\section{B. Clover correction}

Clover fermions are designed to remove $\mathcal{O}(a)$ artifacts arising in the Wilson action. Thus, the focus of this investigation is to determine whether the clover-fermion action removes the $\mathcal{O}(a)$ field-strength-dependent additive mass renormalization due to the Wilson term.

The clover-fermion matrix is given by

$$
D_{\mathrm{cl}}=\not+\frac{a}{2} \Delta-a c_{\mathrm{cl}} \sigma \cdot F+m,
$$

where $a$ is the lattice spacing, $m$ is the bare quark mass, $\nabla$ is the covariant central finite difference operator, $\Delta$ is the lattice Laplacian or Wilson term, and $c_{\mathrm{cl}}$ is the coefficient of the clover term. We define

$$
\sigma \cdot F=\sum_{\mu<\nu} \sigma_{\mu \nu} F_{\mu \nu}
$$

as the clover term (note the sum is restricted to $\mu<\nu$ to avoid double counting), where $\sigma_{\mu \nu}=\frac{i}{2}\left[\gamma_{\mu}, \gamma_{\nu}\right]$ and $F_{\mu \nu}$ is the clover discretization of the lattice field strength tensor. In general, for QCD + QED calculations we need to consider the chromomagnetic- and electromagnetic-field strength contributions separately [12]:

$$
F_{\mu \nu}=F_{\mu \nu}^{\mathrm{qcd}}+F_{\mu \nu}^{\mathrm{em}}
$$

In the free-field case, all the QCD links are set to 1 so the QCD field strength $F_{\mu \nu}^{\mathrm{qcd}}=0$. Here we consider only the electromagnetic-field strength and attempt to determine the appropriate value for the free parameter $c_{\mathrm{cl}}$, which is defined to be the coefficient of the electromagnetic clover term. Setting

$C_{\mu \nu}(x)=\frac{1}{4}\left(P_{\mu, \nu}(x)+P_{\nu,-\mu}(x)+P_{-\nu, \mu}(x)+P_{-\mu,-\nu}(x)\right)$

to be the average of the four elementary plaquettes, it is easy to show that the electromagnetic-field strength tensor is constant in a uniform background field:

$$
\begin{aligned}
F_{\mu \nu} & =\frac{1}{2 i}\left(C_{\mu \nu}-C_{\mu \nu}^{\dagger}\right) \\
\Rightarrow F_{12} & =\frac{1}{2 i}\left(e^{+i a^{2} q B}-e^{-i a^{2} q B}\right) \\
& =\sin a^{2} q B .
\end{aligned}
$$

For convenience we define the lattice magnetic-field strength as

$$
B_{L}=\sin a^{2} q B .
$$

For a magnetic field in the $z$ direction then the only nonzero field strength tensor entry is $F_{12}=B_{L}$ such that $\sigma \cdot F$ becomes diagonal with entries $\mp B_{L} / 2$. The Wilson term is simply the lattice Laplacian, which effectively describes a scalar particle. In the free-field case, this causes a shift in the critical mass given by 


$$
a m_{c}(B) \simeq a m_{c}-a^{2}|q B| / 2,
$$

such that the free-field mass is adjusted by

$$
a m(B) \simeq a m(0)+a^{2}|q B| / 2 .
$$

Hence, in a uniform magnetic field there is a corresponding Landau energy $\sim a^{2}|q B| / 2$.

The magnetic part of the clover term for $O(a)$ improvement of the Wilson matrix is given by

$$
-c_{\mathrm{cl}} \sum_{\mu<\nu} \sigma_{\mu \nu} F_{\mu \nu}=-\frac{c_{\mathrm{cl}}}{2}\left(\begin{array}{cccc}
+B_{L} & 0 & 0 & 0 \\
0 & -B_{L} & 0 & 0 \\
0 & 0 & +B_{L} & 0 \\
0 & 0 & 0 & -B_{L}
\end{array}\right) .
$$

This operator commutes with the lattice Laplacian, and as both are Hermitian it is possible to write down a shared eigenvector basis. The lowest eigenvalue for the clover term in a uniform background field is

$$
\begin{aligned}
\lambda_{\min } & =-c_{\mathrm{cl}}\left|B_{L}\right| / 2 \\
& =-c_{\mathrm{cl}}\left|\sin a^{2} q B\right| / 2 \\
& \simeq-c_{\mathrm{cl}} a^{2}|q B| / 2 .
\end{aligned}
$$

It is clear then that for small field strengths, if we set $c_{\mathrm{cl}}=1$ to the tree-level value, the clover term will cancel the Landau shift induced by the Wilson term.

\section{Tree-level results}

Numerical results for the pion energies in a background magnetic field are shown in Fig. 1 for Wilson fermions and

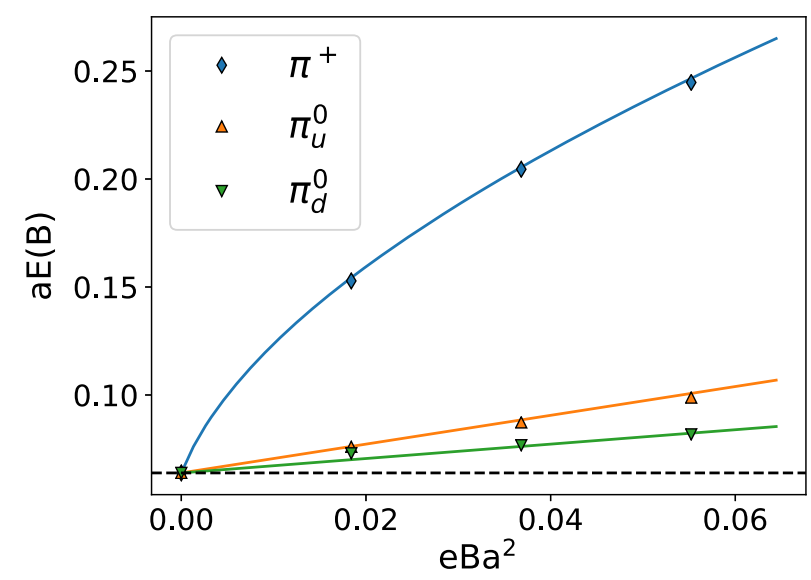

FIG. 1. Pion energies from Wilson-fermion correlation functions as a function of background magnetic-field strength. The colored curves are the expected energies for Wilson fermions based on Eqs. (10) and (11).

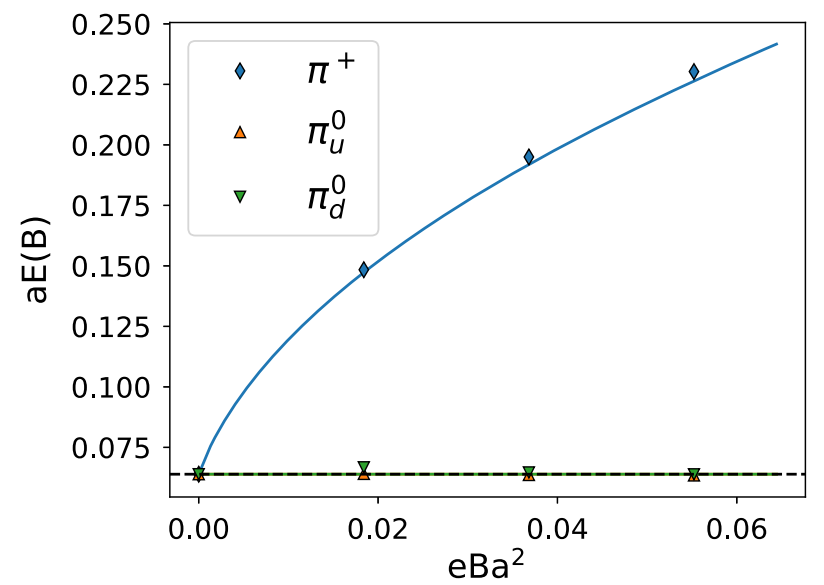

FIG. 2. Pion energies from clover-fermion correlation functions as a function of background magnetic-field strength. The colored lines are the expected energies in the absence of the Wilson background-field additive mass renormalization, constant for the neutral pions and Eq. (12) for the charged pion.

Fig. 2 for clover fermions. Here we have selected a hopping parameter value of $\kappa=0.12400$, relative to the bare critical hopping parameter of $\kappa_{\mathrm{cr}}=1 / 8$, in anticipation of exploring full QCD where the PACS-CS Sommer scale provides $a=0.0951 \mathrm{fm}$. This kappa value corresponds to a pion mass of $140 \mathrm{MeV}$. These calculations are performed on a lattice with antiperiodic boundary conditions in the time direction and fits accommodate both the forward and backward propagating states.

It is clear from Fig. 1 that the neutral-pion energy closely follows that described by Eq. (10). The additive mass renormalization due to the Wilson term is hence correctly described by Eqs. (1) and (10). The charged $\pi^{+}$energy using Wilson fermions agrees with the analytic energy of Eq. (11), further validating the additive mass renormalization of Eq. (1).

The results shown in Fig. 2 for the clover-fermion action verify our calculation for the tree-level coefficient correction. The energies of the two neutral pions $\pi_{d}^{0}$ and $\pi_{u}^{0}$ depicted for clover-improved fermions do not show this additive mass renormalization; they do not change as a function of field strength. Similarly, the charged pion results agree with Eq. (12) for the pion energy in the absence of the Wilson background-field additive mass renormalization. Thus the clover term effectively removes the $\mathcal{O}(a)$ additive mass renormalization of the Wilson term in the background magnetic field.

\section{QCD CASE}

We have demonstrated that the addition of the tree-level clover term in the case of the electromagnetic $U(1)$ field provides a correction that removes the background-fielddependent additive mass renormalization induced by the Wilson term. The next question that arises is whether this 
correction survives once QCD interactions are turned on, and what (if any) adjustments are needed to the clover coefficient.

To study this, we allow for the QCD and electromagnetic-field strengths to have different clover coefficients:

$$
c_{\mathrm{cl}} F_{\mu \nu} \rightarrow c_{\mathrm{sw}} F_{\mu \nu}^{\mathrm{qcd}}+c_{\mathrm{em}} F_{\mu \nu}^{\mathrm{em}} .
$$

Here $c_{\mathrm{sw}}$ is the coefficient of the traditional SheikholeslamiWohlert improvement term arising from the $S U(3)$ QCD gauge field interactions, and $c_{\mathrm{em}}$ is the coefficient of the clover term induced by the $U(1)$ electromagnetic gauge field.

We know that the $c_{\mathrm{sw}}$ coefficient is renormalized by QCD. In particular, here we will be studying clover fermions with a nonperturbatively improved (QCD) coefficient. To determine the appropriate value for $c_{\mathrm{em}}$ in this instance, we will first consider the "naive" case and set $c_{\mathrm{em}}=c_{\mathrm{sw}}$ such that the clover terms from the QCD and electromagnetic-field strengths are treated in a uniform way. Having done this, we will look for the presence of magnetic-field-strength-dependent artifacts in the pion mass, as was done for the QCD free-field case.

Once QCD interactions are present, the pion possesses an internal structure and mass which is dependent on the mass of the quarks in a more subtle way $[16,17]$ than in Eqs. (10) and (11). There is now a complex interplay between the background field and QCD effects $[9,18]$.

The energy of a relativistic pion with mass $m_{\pi}$ and charge $q e$ in a magnetic field orientated in the $\hat{z}$ direction is

$$
\begin{aligned}
E_{\pi, n}^{2}(B)= & m_{\pi}^{2}+(2 n+1)|q e B|+p_{z}^{2} \\
& -4 \pi m_{\pi} \beta_{\pi} B^{2}+\mathcal{O}\left(B^{3}\right),
\end{aligned}
$$

where $\beta_{\pi}$ is the magnetic polarizability, which is chargestate dependent. It is necessary to use this fully relativistic form of the energy for the pion rather than the Taylor expansion common in the literature [2,4-6,9] as $2 m /(E+m)$ differs substantially from one for the largest field strength [9]. At $m_{\pi}=296 \mathrm{MeV}$ this difference is as much as $22 \%$ and is significant when working with the increased precision of pion correlation functions.

\section{A. Simulation details}

This work considers the $2+1$ flavor dynamical gauge configurations provided by the PACS-CS [19] Collaboration via the International Lattice Data Grid (ILDG) [20]. These configurations are founded on a nonperturbatively improved clover-fermion action and Iwasaki gauge action [21]. Two values of the light quark hopping parameter $\kappa_{u d}=0.13754,0.13770$ are considered corresponding to pion masses $m_{\pi}=411$ and $296 \mathrm{MeV}$ [19]. The lattice spacing for each mass is set using the Sommer scale [19] with $r_{0}=0.49 \mathrm{fm}$. The lattice volume is $L^{3} \times T=$ $32^{3} \times 64$ and the ensemble sizes are 449 and 400 configurations, respectively. Unless otherwise mentioned, we focus on results from the lighter ensemble with $\kappa_{u d}=$ 0.13770 and $m_{\pi}=296 \mathrm{MeV}$.

Two-point correlation functions at three distinct nonzero background magnetic-field strengths are calculated. To do this, propagators at ten nonzero field strengths are created. With reference to Eq. (6), the field strengths considered have $k_{B}= \pm 1, \pm 2, \pm 3, \pm 4$, and \pm 6 in Eq. (6). These give physical field strengths of $e B= \pm 0.087, \pm 0.174, \pm 0.261$, \pm 0.348 , and $\pm 0.522 \mathrm{GeV}^{2}$, respectively. Correlation functions are averaged over both positive and negative field strengths during analysis to provide an improved unbiased estimator.

We note that these configurations are electroquenched; the field exists only for the valence quarks of the hadron. To include the background field at configuration generation time is possible [22] but requires a separate hybrid Monte Carlo calculation for each field strength, which is prohibitively expensive. Fortunately, these effects are not relevant to the current investigation.

Three-dimensional spatial Gaussian smearing utilizing stout-smeared links is applied at the source and a point sink is considered. This ensures that the pion ground state is well represented. Standard pseudoscalar interpolating fields $\chi=\bar{q} \gamma_{5} q$ are used, where the quark flavors are either $u \bar{u}$ or $\overline{d \bar{d}}$ corresponding to $\pi_{u}^{0}$ and $\pi_{d}^{0}$.

\section{B. Energy shifts for Wilson fermions}

To investigate the effect of the nonperturbatively improved clover coefficient in the full QCD calculations, the lowest-lying neutral-pion background-field energy is considered:

$$
E_{\pi^{0}}^{2}(B)=m_{\pi^{0}}^{2}-4 \pi m_{\pi^{0}} \beta_{\pi^{0}} B^{2}+\mathcal{O}\left(B^{3}\right) .
$$

To determine how the known additive quark-mass renormalization of Eq. (1) effects the pion mass in full QCD, we commence with the consideration of the Gell-Mann-OakesRenner relation $[16,23]$

$$
\begin{aligned}
m_{\pi^{0}}^{2} & =-\frac{2 m_{u / d}}{f_{\pi}^{2}}\langle\Omega|\bar{u} u| \Omega\rangle \\
& \equiv m_{u / d} E_{\Omega},
\end{aligned}
$$

where $E_{\Omega}=-2\langle\Omega|\bar{u} u| \Omega\rangle / f_{\pi}^{2}, f_{\pi}$ is the pion decay constant and $\langle\Omega|\bar{u} u| \Omega\rangle$ is the chiral condensate. As $m_{\pi}^{2} \propto m_{q}, E_{\Omega}$ has a relatively weak quark-mass dependence.

Using Eq. (1), we introduce a background-fielddependent pion mass due to the Wilson additive mass renormalization:

$$
\begin{aligned}
m_{\left[\pi^{0}\right]}^{2}(B) & =m_{[u / d]}(B) E_{\Omega}(B) \\
& =\left(m_{u / d}+\frac{a \xi}{2}\left|q_{u / d} e B\right|\right) E_{\Omega}(B) .
\end{aligned}
$$


Because QCD effects will modify Eq. (1), we have introduced a coefficient $\xi$ in Eq. (27) which in principle can be $B$-field dependent.

For example, Bali et al. [12] investigated the change in the quark mass in Wilson-fermion QCD plus background magnetic-field simulations by examining the change in the critical hopping parameter as a function of magnetic-field strength. For small external magnetic-field strengths the mass shift is an order of magnitude smaller from the freefield case and for their smallest field strengths, the sign of the shift differs. The dependence of Eq. (1) begins to emerge at large magnetic-field strengths as QCD effects become small. As discussed in the following subsection, our nonperturbatively improved clover-fermion results also display this order of magnitude suppression. However our survey of magnetic-field strengths does not reveal the Wilson-fermion sign change in the mass shift. Thus it is sufficient to treat $\xi$ as a constant to be determined.

Studies [24,25] have indicated that $E_{\Omega}$ changes slowly in an external magnetic field and on this basis, we consider the leading-order approximation

$$
\begin{aligned}
m_{\left[\pi^{0}\right]}^{2}(B) & \simeq\left(m_{u / d}+\frac{a \xi}{2}\left|q_{u / d} e B\right|\right) E_{\Omega}(0) \\
& \simeq m_{\pi^{0}}^{2}+\frac{a \xi}{2}\left|q_{u / d} e B\right| E_{\Omega}(0)
\end{aligned}
$$

Finally the energy of a neutral pion in an external magnetic field using Wilson fermions is

$$
\begin{aligned}
E_{\left[\pi^{0}\right]}^{2}(B)= & m_{\left[\pi^{0}\right]}^{2}-4 \pi m_{\left[\pi^{0}\right]} \beta_{\pi^{0}} B^{2}+\mathcal{O}\left(B^{3}\right), \\
\simeq & m_{\pi^{0}}^{2}+\frac{a \xi}{2} E_{\Omega}(0)\left|q_{u / d} e B\right| \\
& -4 \pi \beta_{\pi^{0}} B^{2} \sqrt{m_{\pi^{0}}^{2}+\frac{a \xi}{2} E_{\Omega}(0)\left|q_{u / d} e B\right| .}
\end{aligned}
$$

We note that terms linear in $B$ from the magnetic-field dependence of $E_{\Omega}$ in Eq. (27) can combine with the linear term $\frac{a \xi}{2}\left|q_{u / d} e B\right|$ to provide a contribution proportional to $B^{2}$, thus contaminating the $\mathcal{O}\left(B^{2}\right)$ signal used to extract $\beta_{\pi^{0}}$. Ultimately, it is important to ensure this $\mathcal{O}(a)$ term is removed.

To explore the presence of additive quark-mass renormalization, we focus on the quantity

$$
\begin{aligned}
& E_{\left[\pi^{0}\right]}^{2}(B)-m_{\pi^{0}}^{2} \simeq \frac{a \xi}{2} E_{\Omega}(0)\left|q_{u / d} e B\right| \\
& -4 \pi \beta_{\pi^{0}} B^{2} \sqrt{m_{\pi^{0}}^{2}+\frac{a \xi}{2} E_{\Omega}(0)\left|q_{u / d} e B\right| .}
\end{aligned}
$$

This energy shift can be constructed using the two correlator combinations

$$
\begin{gathered}
R_{+}(B, t)=G(B, t) G(0, t), \\
R_{-}(B, t)=\frac{G(B, t)}{G(0, t)},
\end{gathered}
$$

where $G(B, t)$ is the zero-momentum projected two-point correlation function. Upon taking the effective energy

$$
E_{\text {eff }}(t)=\frac{1}{\delta t} \log \left(\frac{G(t)}{G(t+\delta t)}\right),
$$

Eq. (31) yields $\left(E_{\left[\pi^{0}\right]}(B)+m_{\pi^{0}}\right)$ and Eq. (32) provides $\left(E_{\left[\pi^{0}\right]}(B)-m_{\pi^{0}}\right)$. These effective-energy shifts from $R_{+}$ and $R_{-}$are then multiplied together to form the $E_{\left[\pi^{0}\right]}^{2}(B)-$ $m_{\pi^{0}}^{2}$ energy shift of Eq. (30). We note that Eq. (32) is particularly helpful in isolating $B$-dependent terms, as QCD contributions are correlated in the ratio of correlation functions and largely cancel.

Noting Eq. (30) has leading linear and quadratic terms in $B$ we commence by considering the fit function

$$
E_{\left[\pi^{0}\right]}^{2}(B)-m_{\pi^{0}}^{2}=c_{1} k_{B}+c_{2} k_{B}^{2} .
$$

Here $k_{B}$ is the quantization number from the quantization condition on magnetic-field strength of Eq. (6). An estimate for the fit parameter $c_{1}$ can be obtained from Eq. (30):

$$
c_{1}=\frac{\pi}{a}\left|\frac{q_{u / d}}{q_{d}}\right| \frac{\xi E_{\Omega}(0)}{N_{x} N_{y}} .
$$

Recalling the Gell-Mann-Oakes-Renner relation at zero magnetic field provides $E_{\Omega}(0)=m_{\pi^{0}}^{2} / m_{q}$ and drawing on the Wilson quark-mass relation

$$
m_{q}=\frac{1}{2 a}\left(\frac{1}{\kappa}-\frac{1}{\kappa_{\mathrm{cr}}}\right)
$$

where $\kappa_{\mathrm{cr}}$ is the critical hopping parameter where the zerofield pion mass vanishes, Eq. (35) can be written

$$
c_{1}=2 \pi\left|\frac{q_{u / d}}{q_{d}}\right| \frac{\xi m_{\pi^{0}}^{2}}{N_{x} N_{y}}\left(\frac{1}{\kappa}-\frac{1}{\kappa_{\mathrm{cr}}}\right)^{-1} .
$$

Similarly, drawing on Eq. (30), $c_{2}$ is related to the magnetic polarizability

$$
\beta=-c_{2} \alpha \frac{q_{d}^{2} a^{4}}{m_{\left[\pi^{0}\right]}}\left(\frac{N_{x} N_{y}}{2 \pi}\right)^{2},
$$

where $m_{\left[\pi^{0}\right]}$ is provided in Eq. (28) and $\alpha=1 / 137.036$ is the fine structure constant. Of course, if $c_{1} \neq 0$, then the magnetic-field dependence of $E_{\Omega}$ in Eq. (35) will induce additional $\mathcal{O}\left(B^{2}\right)$ contaminations to Eq. (38). Thus it is vital to remove this $\mathcal{O}(a)$ error. 

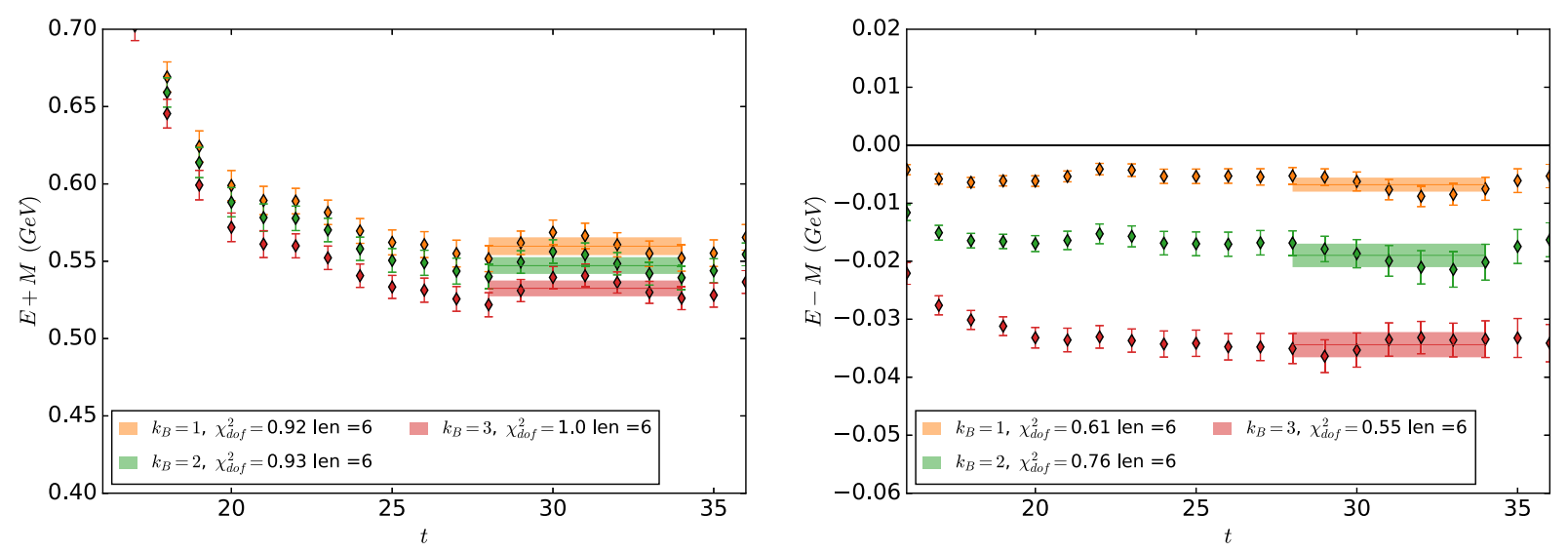

FIG. 3. Neutral-pion energy shifts from Eqs. (31) and (32) for $E_{\left[\pi^{0}\right]}(B)+m_{\pi^{0}}$ (left) and $E_{\left[\pi^{0}\right]}(B)-m_{\pi^{0}}$ (right), respectively, using a nonperturbatively improved clover-fermion action on the $m_{\pi}=296 \mathrm{MeV}$ ensemble. The three smallest field strengths are illustrated. Shaded regions illustrate the fit windows selected through the consideration of the full covariance matrix $\chi_{\text {dof }}^{2}$, the extent of the fit window and the desire to select the same fit window for all effective-energy shifts.

As the $u$ and $d$ quarks are mass degenerate in our lattice QCD simulation, the neutral-pion correlation functions with quark content $u \bar{u}$ or $d \bar{d}$ differ only by the strength of the quark flavor interactions with the external magnetic field. This allows the neutral-pion correlation function to be evaluated at a larger range of field strengths than is possible for the $\pi^{+}$, using the same propagators.

\section{Nonperturbatively improved clover fermions}

The nonperturbatively improved clover-fermion action used in the full QCD calculations differs from that used in the free-field calculations by the value of the clover coefficient $c_{\mathrm{sw}}$ multiplying the $\mathcal{O}(a)$ clover term of the fermion action in Eq. (13).

The free-field simulations used the tree-level value $c_{\mathrm{sw}}=1$ while the full QCD calculations use the nonperturbatively improved value $c_{\mathrm{sw}}=1.715[19,26]$. The extent to which this changes the cancellation of the additive mass renormalization seen to occur in Fig. 2 is investigated using the energy shift defined above in Eq. (30).

The $E_{\left[\pi^{0}\right]}(B)-m_{\pi^{0}}$ energy shift is illustrated in Fig. 3, right; it is quite clear that it is easy to construct good plateau fits for this energy shift. This is in contrast to the $E_{\left[\pi^{0}\right]}(B)+$ $m_{\pi^{0}}$ energy shift in Fig. 3, left, as the correlated QCD fluctuations between field strengths compound rather than cancel, making it difficult to fit constant plateaus. This difficulty in fitting constant plateaus reduces the fit parameter space considered as common plateau fits are required for both $E_{\left[\pi^{0}\right]}(B)-m_{\pi^{0}}$ and $E_{\left[\pi^{0}\right]}(B)+m_{\pi^{0}}$. The fit window $t=[28,34]$ is chosen as this window has good fits with acceptable $\chi_{\text {dof }}^{2}$ 's across each field strength and energy-shift type considered.

The energy shifts are fitted as a function of the fieldstrength quanta $k_{B}$ in Fig. 4. Recalling Eq. (30) has leading linear and quadratic terms in $B$ we consider the fit function

$$
E_{\left[\pi^{0}\right]}^{2}(B)-m_{\pi^{0}}^{2}=c_{1} k_{B}+c_{2} k_{B}^{2}
$$

Under the assumption of the removal of additive mass renormalization, we first consider fixing $c_{1}=0$ and using a $c_{2} k_{B}^{2}$ quadratic-only fit function. As illustrated in Fig. 4 this provides a poor description of the results and yields an unacceptable $\chi_{\text {dof }}^{2}=6.1$.

Allowing for a nontrivial $c_{1}$ coefficient provides the linear + quadratic, $c_{1} k_{B}+c_{2} k_{B}^{2}$ fit of Eq. (39). This fit describes the lattice simulation results well with a $\chi_{\mathrm{dof}}^{2}=0.5$. However, it also indicates the presence of additive mass renormalization in the nonperturbatively improved clover-fermion simulation.

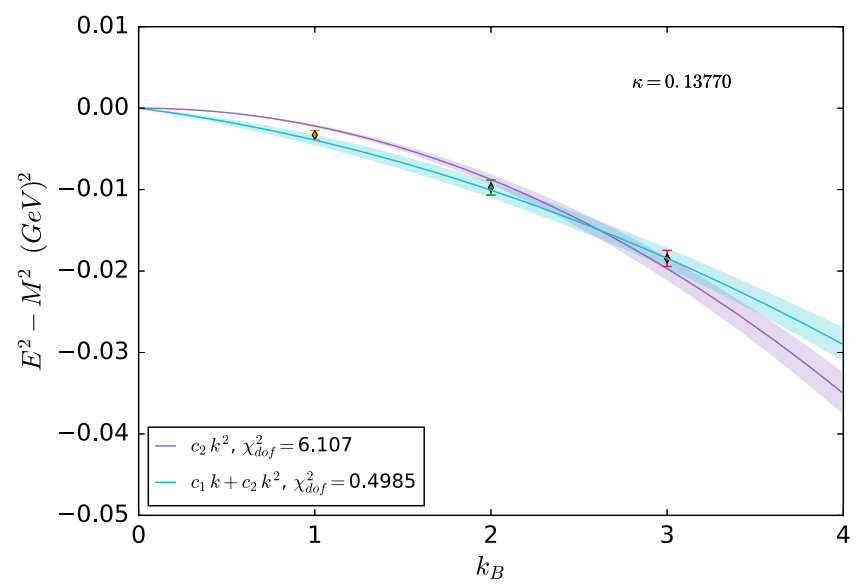

FIG. 4. Fits of the magnetic-field-induced energy shift to the magnetic-field quanta for the nonperturbatively improved cloverfermion action. The full covariance-matrix-based $\chi_{\text {dof }}^{2}$ provides evidence of a nontrivial value for fit coefficient $c_{1}$, indicating the presence of unwanted Wilson-like additive mass renormalizations in the nonperturbatively improved clover-fermion action. 

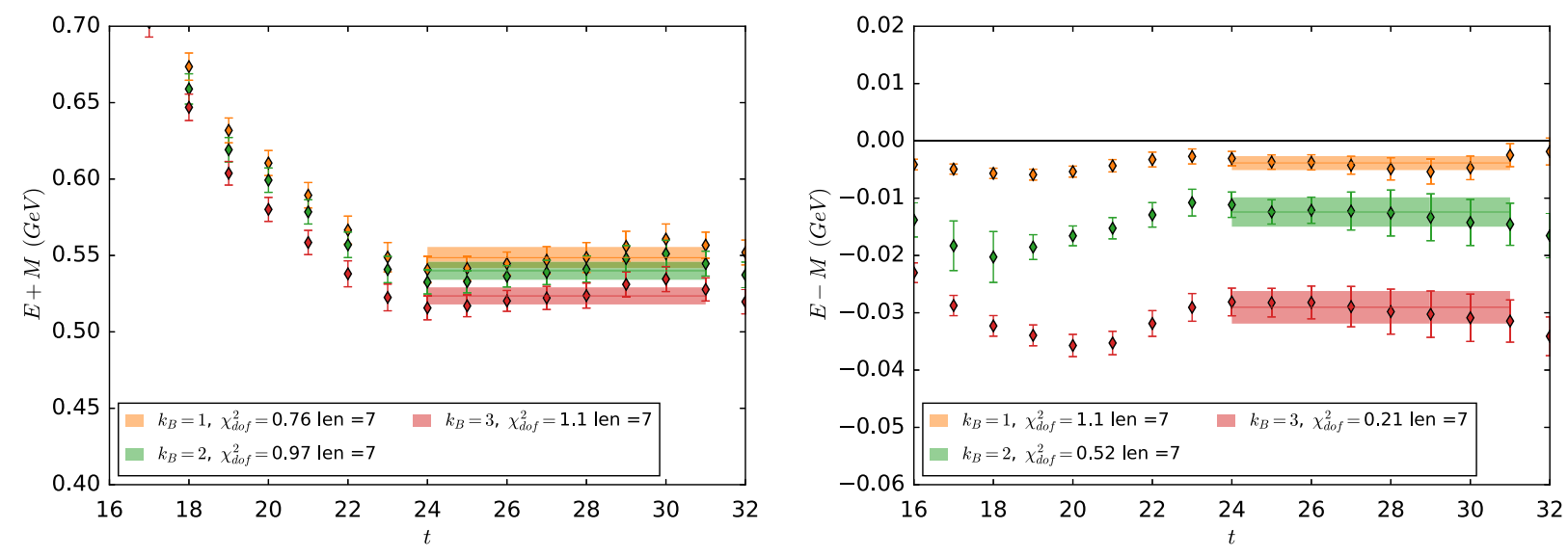

FIG. 5. Neutral-pion energy shifts from Eqs. (31) and (32) for $E_{\pi^{0}}(B)+m_{\pi^{0}}$ (left) and $E_{\pi^{0}}(B)-m_{\pi^{0}}$ (right), respectively, using the BFC clover-fermion action on the $m_{\pi}=296 \mathrm{MeV}$ ensemble. The three smallest field strengths are illustrated. Shaded regions illustrate the fit windows selected through the consideration of the full covariance matrix $\chi_{\text {dof }}^{2}$, the extent of the fit window and the desire to select the same fit window for all effective-energy shifts.

To explore the necessity of this $\mathcal{O}(a)$ term linear in the magnetic-field strength, a quadratic + cubic, $c_{2} k_{B}^{2}+c_{3} k_{B}^{3}$, fit is also considered. However, this model does not describe the simulation results, producing unacceptably high $\chi_{\text {dof }}^{2}$ values. Fits over four field strengths are also considered. This time, the acceptable fit requires linear, quadratic and cubic terms. Thus, the demand for an $\mathcal{O}(a)$ term associated with the presence of additive mass renormalization is robust.

\section{Expected mass renormalization}

These results indicate that the current treatment of the clover term is inadequate. In light of the success observed in the free-field limit, we anticipate that the application of the nonperturbatively improved value $c_{\mathrm{sw}}^{\mathrm{NP}}=1.715$ to both the QCD and the background-field contributions to the clover term has spoiled the removal of additive mass renormalization associated with the magnetic field.

If the tree-level value of $c_{\mathrm{sw}}^{\mathrm{tree}}=1$ is the value required for the removal of $\mathcal{O}(a)$ errors, then we have overcompensated by an amount

$$
D^{\mathrm{NP}}=c_{\mathrm{sw}}^{\mathrm{tree}}-c_{\mathrm{sw}}^{\mathrm{NP}}=1.0-1.715=-0.715 .
$$

This overcompensation is relative to the standard Wilson action discrepancy of

$$
D^{\mathrm{W}}=c_{\mathrm{sW}}^{\mathrm{tree}}=1.0 .
$$

This overcompensation factor can be incorporated into the Wilson-fermion discussion of Sec. IV C through

$$
\xi \rightarrow \xi^{\mathrm{NP}}=\frac{D^{\mathrm{NP}}}{D^{\mathrm{W}}} \xi,
$$

enabling a prediction of the nontrivial value for $c_{1}$. Including the aforementioned order of magnitude suppression factor $\xi=1 / 10$, Eqs. (37) and (42) provide an estimate for the fit parameter $c_{1}$. For the $\pi_{d}^{0}$ with $m_{\pi}=296 \mathrm{MeV}, \kappa_{d}=0.13770$ and $\kappa_{\mathrm{cr}}=0.13791$ [19], $c_{1} \sim-3 \times 10^{-3} \mathrm{GeV}^{2}$. From the linear + quadratic $c_{1} k_{B}+$ $c_{2} k_{B}^{2}$ fit, $c_{1}=-2.8(9) \times 10^{-3} \mathrm{GeV}^{2}$.

\section{BACKGROUND-FIELD CORRECTION}

Motivated by the analytic calculation in Sec. III B and encouraged by the above result, in this section we investigate a new clover-fermion action where the coefficients for the QCD and background magnetic-field contributions to the field-strength tensor in Eq. (23) take different values. The electromagnetic clover coefficient takes the tree-level value $c_{\mathrm{em}}=1$, while the QCD clover coefficient retains its nonperturbative value of $c_{\mathrm{sw}}^{\mathrm{NP}}=1.715$. In the following, we refer to this modified fermion action as the backgroundfield-corrected (BFC) form of the clover-fermion action.

\section{A. Additive mass renormalization}

The process of calculating correlation functions and forming energy shifts is repeated as detailed above in Eqs. (31)-(33). Figure 5 displays the new effective energy shifts and the associated fits.

In the absence of additive mass renormalizations, Eq. (30) simplifies to

$$
E_{\pi^{0}}^{2}(B)-m_{\pi^{0}}^{2}=-4 \pi m_{\pi^{0}} \beta_{\pi^{0}} B^{2}+\mathcal{O}\left(B^{3}\right),
$$

and therefore we consider fit functions of the form

$$
E_{\pi^{0}}^{2}(B)-m_{\pi^{0}}^{2}=c_{1} k_{B}+c_{2} k_{B}^{2}+c_{3} k_{B}^{3} .
$$

If the modified clover action has removed the Wilson additive mass renormalization, the linear term of Eq. (44) 


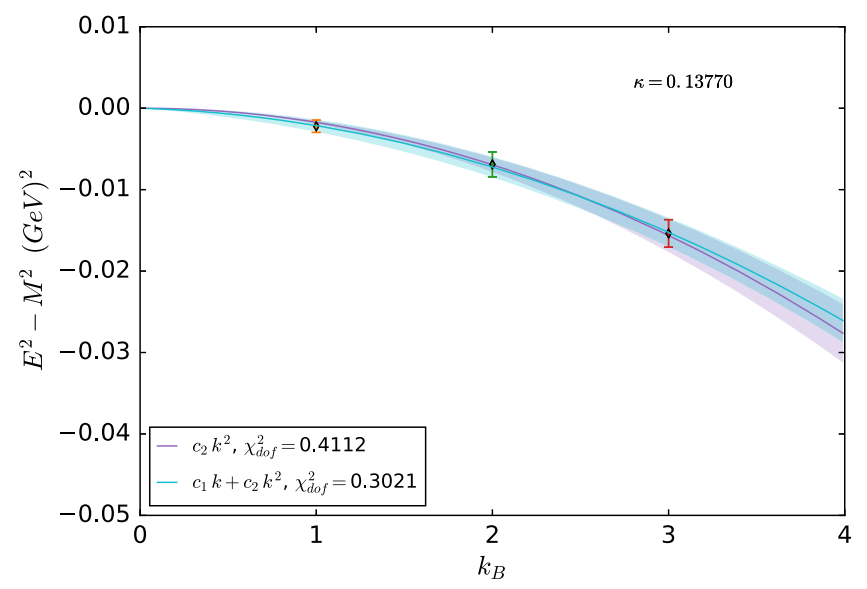

FIG. 6. Fits of the magnetic-field-induced energy shift to the magnetic-field quanta for the BFC clover action. The full covariance-matrix-based $\chi_{\text {dof }}^{2}$ for the simple $c_{2} k_{B}^{2}$ quadratic fit provides evidence of the elimination of Wilson-like additive mass renormalizations in the BFC clover action. Allowing for a nontrivial value of $c_{1}$ produces a value consistent with zero.

will have a trivial coefficient. Indeed, fits without the linear term should describe the energy shifts well.

Fits of Eq. (44) to results from the lowest three magneticfield strengths are illustrated in Fig. 6. As acceptable fits are obtained with the two leading terms, $c_{3}$ has been constrained to zero. The success of our BFC clover-fermion action is reflected in the excellent description of the results using only a quadratic term in Eq. (44), in accord with the expectations of Eq. (43). Allowing for the possibility of a nontrivial value for $c_{1}$, we find $c_{1}=(-5.3 \pm 8.7) \times$ $10^{-4} \mathrm{GeV}^{2}$, consistent with zero.

Drawing on the full range of five magnetic-field strengths available to $d \bar{d}$ and $u \bar{u}$ pions, Fig. 7 illustrates fits of Eq. (44) to the BFC clover results. This time the

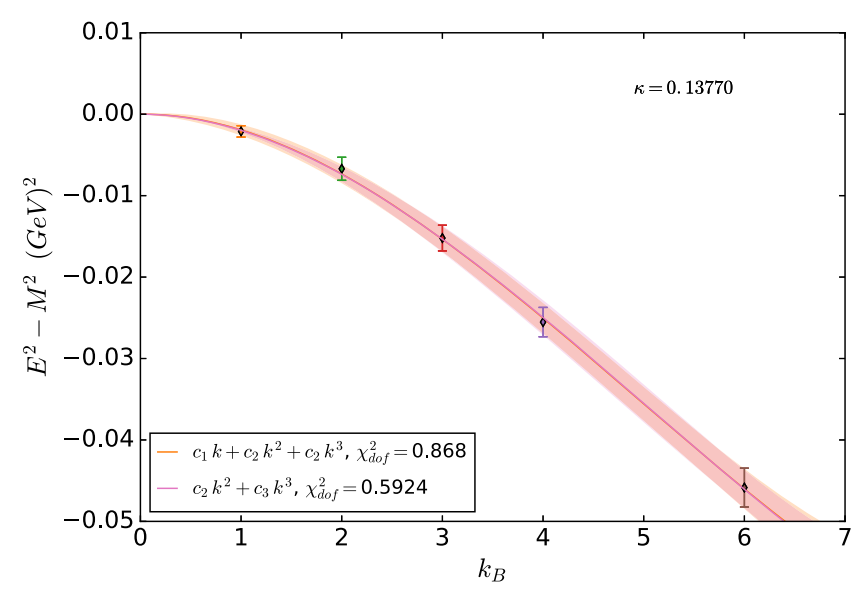

FIG. 7. Fits of the magnetic-field-induced energy shift to the first five magnetic-field quanta for the BFC clover action. Here a term cubic in the magnetic-field strength is required to describe the largest field strength. results demand $c_{3} \neq 0$ and thus a $B^{3}$ term is manifest. Again, the success of our BFC clover action in removing additive mass renormalizations is reflected in the excellent description of the results using only quadratic and cubic terms in Eq. (44), in accord with the expectations of Eq. (43). Allowing for the possibility of a nontrivial value for $c_{1}$, we find $c_{1}=(1.1 \pm 9.0) \times 10^{-4} \mathrm{GeV}^{2}$, consistent with zero.

We note that similar results are observed for fits to the first four magnetic-field strengths. The lattice results are described well by fits with $c_{1}$ constrained to zero, and allowing for a nontrivial value provides $c_{1}=$ $(-6.2 \pm 8.2) \times 10^{-4} \mathrm{GeV}^{2}$, again consistent with zero. While it is sufficient to set $c_{3}=0$, the onset of nontrivial $\mathcal{O}\left(B^{3}\right)$ terms lies between the fourth and fifth field strengths and, therefore, only the three smallest field strengths are considered in determining the magnetic polarizability.

In summary, the BFC clover-fermion action has successfully removed the additive quark-mass renormalization due to the Wilson term. The key is to employ the tree-level value $c_{\mathrm{em}}=1$ for uniform background-field contributions to the electromagnetic clover term.

\section{B. Magnetic polarizability}

Having demonstrated the removal of additive quarkmass renormalization in the BFC clover action, the magnetic polarizability can be determined without concern for the aforementioned $\mathcal{O}(a)$ contaminations entering the $B^{2}$ associated with the magnetic polarizability.

The energy shift and fits performed in Sec. VA are used and the polarizability extracted from the coefficient $c_{2}$ of the quadratic term of Eq. (44). Comparing with Eq. (43) and drawing on the field quantization condition of Eq. (5) with $q=q_{d}=-1 / 3$, the magnetic polarizability is given by

$$
\beta=-c_{2} \alpha \frac{q_{d}^{2} a^{4}}{m_{\pi}}\left(\frac{N_{x} N_{y}}{2 \pi}\right)^{2}
$$

Results for the magnetic polarizability of the neutral pion from the $\mathcal{O}(a)$-improved BFC clover action analysis of the lowest three magnetic-field strengths are reported in Table I. While the report of our analysis has focused on the light-quark hopping parameter of $\kappa_{u d}=0.13770$ corresponding to $296 \mathrm{MeV}$, results for $m_{\pi}=411$ are also reported.

TABLE I. Magnetic polarizability of the neutral pion from the $\mathcal{O}(a)$-improved BFC clover action analysis of the lowest three magnetic-field strengths.

\begin{tabular}{lcc}
\hline \hline$\kappa$ & $m_{\pi}(\mathrm{MeV})$ & $\beta_{\pi^{0}}\left(\times 10^{-4} \mathrm{fm}^{3}\right)$ \\
\hline 0.13754 & 411 & $0.62(4)$ \\
0.13770 & 296 & $0.54(7)$ \\
\hline \hline
\end{tabular}


As discussed in the previous section, we consider the first three field strengths to avoid the possibility of complications associated with nontrivial $\mathcal{O}\left(B^{3}\right)$ contributions. While the inclusion of a fourth point takes the magnetic polarizability from $\beta_{\pi^{0}}=0.54(7)$ to $0.52(5) \times 10^{-4} \mathrm{fm}^{3}$, the small improvement in statistical uncertainty may be offset by an increase in systematic uncertainty associated with nontrivial $\mathcal{O}\left(B^{3}\right)$ contributions.

\section{CONCLUSIONS}

In this paper the response of the pion to a uniform background magnetic field has been investigated. The existence of a field-strength-dependent additive quark-mass renormalization associated with Wilson fermions was confirmed. We performed an analytic calculation showing that at tree level in the QCD-free case the clover term corrects for these spurious contributions.

When QCD interactions are included, a careful treatment of the QCD and electromagnetic clover terms in Eq. (23) is required. While the interactions of QCD require the nonperturbatively improved value of $c_{\mathrm{sw}}=1.715$, it is essential to apply the tree-level value $c_{\mathrm{sw}}=1$ to the background magnetic-field contributions. With this treatment, it is possible to simultaneously remove the $\mathcal{O}(a)$ errors in the QCD contributions and correct for the $\mathcal{O}(a)$ errors induced by the Wilson term in a background magnetic field. We refer to this modified fermion action as the backgroundfield-corrected clover-fermion action.

With the suppression of $\mathcal{O}(a)$ errors associated with additive mass renormalization in the BFC clover action, the magnetic polarizability of the neutral pion can now been determined. For the first time, a fully relativistic approach to the energy shift is used. Results are summarized in Table I and we anticipate these results will facilitate consensus within the field. $\beta_{\pi^{0}}$ is positive, such that the energy of a pion in a magnetic field decreases. The extent to which this trend continues is of interest and our results give a hint that the $\mathcal{O}\left(B^{3}\right)$ contributions soften this trend.

Future work will approach the physical quark-mass regime and interface with chiral perturbation theory studies [27]. It will also be important to directly include sea-quarkloop interactions with the background magnetic field to incorporate these contributions to the magnetic polarizability. However, this approach is prohibitively expensive. It requires a separate Monte Carlo ensemble for each field strength considered and this will result in a loss of important QCD correlations between different magneticfield strengths.

An alternative approach is to separate the valence and sea-quark-loop contributions to the magnetic polarizability in effective field theory [7,9]. This will enable an accurate extrapolation of current lattice QCD results and an estimation of sea-quark-loop contributions to the magnetic polarizabilities of the pion.

\section{ACKNOWLEDGMENTS}

We thank the PACS-CS Collaboration for making their $2+1$ flavor configurations available and the ongoing support of the ILDG. This work was supported with supercomputing resources provided by the Phoenix HPC service at the University of Adelaide. This research was undertaken with the assistance of resources from the National Computational Infrastructure (NCI). NCI resources were provided through the National Computational Merit Allocation Scheme, supported by the Australian Government through Grants No. LE190100021 and No. LE160100051 and the University of Adelaide Partner Share. R. B. was supported by an Australian Government Research Training Program Scholarship. This research is supported by the Australian Research Council through Grants No. DP140103067, No. DP150103164, No. DP190102215 (D. L.) and No. DP190100297 (W. K.).
[1] J. Smit and J. C. Vink, Nucl. Phys. B286, 485 (1987).

[2] G. Martinelli, G. Parisi, R. Petronzio, and F. Rapuano, Phys. Lett. 116B, 434 (1982).

[3] M. Burkardt, D. B. Leinweber, and X.-m. Jin, Phys. Lett. B 385, 52 (1996).

[4] B. C. Tiburzi and S. O. Vayl, Phys. Rev. D 87, 054507 (2013).

[5] C. W. Bernard, T. Draper, K. Olynyk, and M. Rushton, Phys. Rev. Lett. 49, 1076 (1982).

[6] T. Primer, W. Kamleh, D. Leinweber, and M. Burkardt, Phys. Rev. D 89, 034508 (2014).

[7] J. M. M. Hall, D. B. Leinweber, and R. D. Young, Phys. Rev. D 89, 054511 (2014).
[8] E. V. Luschevskaya, O. E. Solovjeva, O. A. Kochetkov, and O. V. Teryaev, Nucl. Phys. B898, 627 (2015).

[9] R. Bignell, J. Hall, W. Kamleh, D. Leinweber, and M. Burkardt, Phys. Rev. D 98, 034504 (2018).

[10] D. S. Roberts, P. O. Bowman, W. Kamleh, and D. B. Leinweber, Phys. Rev. D 83, 094504 (2011).

[11] B. B. Brandt, G. Bali, G. Endrödi, and B. Glässle, Proc. Sci., LATTICE2015 (2016) 265.

[12] G. S. Bali, B. B. Brandt, G. Endrodi, and B. Glassle, Phys. Rev. D 97, 034505 (2018).

[13] P. H. Ginsparg and K. G. Wilson, Phys. Rev. D 25, 2649 (1982).

[14] H. Neuberger, Phys. Lett. B 417, 141 (1998). 
[15] B. Sheikholeslami and R. Wohlert, Nucl. Phys. B259, 572 (1985).

[16] M. Gell-Mann, R. J. Oakes, and B. Renner, Phys. Rev. 175, 2195 (1968).

[17] J. Gasser and H. Leutwyler, Phys. Rep. 87, 77 (1982).

[18] F. Bruckmann, G. Endrodi, M. Giordano, S. D. Katz, T. G. Kovacs, F. Pittler, and J. Wellnhofer, Phys. Rev. D 96, 074506 (2017).

[19] S. Aoki, K. I. Ishikawa, N. Ishizuka, T. Izubuchi, D. Kadoh, K. Kanaya, Y. Kuramashi, Y. Namekawa, M. Okawa, Y. Taniguchi, A. Ukawa, N. Ukita, and T. Yoshie (PACS-CS Collaboration), Phys. Rev. D 79, 034503 (2009).

[20] M. G. Beckett, B. Joo, C. M. Maynard, D. Pleiter, O. Tatebe, and T. Yoshie, Comput. Phys. Commun. 182, 1208 (2011).

[21] Y. Iwasaki, arXiv:1111.7054.
[22] H. R. Fiebig, W. Wilcox, and R. M. Woloshyn, Nucl. Phys. B324, 47 (1989).

[23] C. Gattringer and C. B. Lang, Lect. Notes Phys. 788, 1 (2010).

[24] M. D’Elia and F. Negro, Phys. Rev. D 83, 114028 (2011).

[25] G. S. Bali, F. Bruckmann, G. Endrodi, Z. Fodor, S. D. Katz, S. Krieg, A. Schafer, and K. K. Szabo, J. High Energy Phys. 02 (2012) 044.

[26] S. Aoki, M. Fukugita, S. Hashimoto, K. I. Ishikawa, N. Ishizuka, Y. Iwasaki, K. Kanaya, T. Kaneko, Y. Kuramashi, M. Okawa, S. Takeda, Y. Taniguchi, N. Tsutsui, A. Ukawa, N. Yamada, and T. Yoshie (JLQCD and CP-PACS Collaborations), Phys. Rev. D 73, 034501 (2006).

[27] S. Bellucci, J. Gasser, and M. E. Sainio, Nucl. Phys. B423, 80 (1994); B431, 413 (1994). 